\title{
Totaltrack VLM vs. Macintosh laryngoscope for orotracheal intubation in obese patients (OVESION RCT)
}

\begin{abstract}
E. Martinez-Hurtado 1, M. Mariscal Flores 2, M. Sanchez-Merchante 3, S. D. Bergese 4, G. Navarro 5, M. Á. Gómez-Ríos 6, AnestesiaR alrway Review Group (AiR Group)

1 Consultant Anaesthetist in Infanta Leonor University Hospital. Anesthesia and Intensive care department. Madrid. Spain

2 Consultant Anaesthetist in Getafe University Hospital. Anesthesia and intensive care department. Madrid. Spain

3 Consultant Anaesthetist in Alcorcon Foundation University Hospital, Madrid. Spain. Anesthesia and intensive care department. Madrid. Spain

4 Clinical Faculty - Wexner Medical Center at The Ohio State University. Ohio (United States)

5 Medical Anaesthesiologist. Anaesthesia and Resuscitation Unit at the Hospital de Emergencias "Dr. Clemente Álvarez" City of Rosario. Province of Santa Fe. Argentina. Santa Fe. Argentina

6 Consultant Anesthesiologist at Complejo Hospitalario Universitario de A Coruña. SERGAS. A Coruña. Spain
\end{abstract}

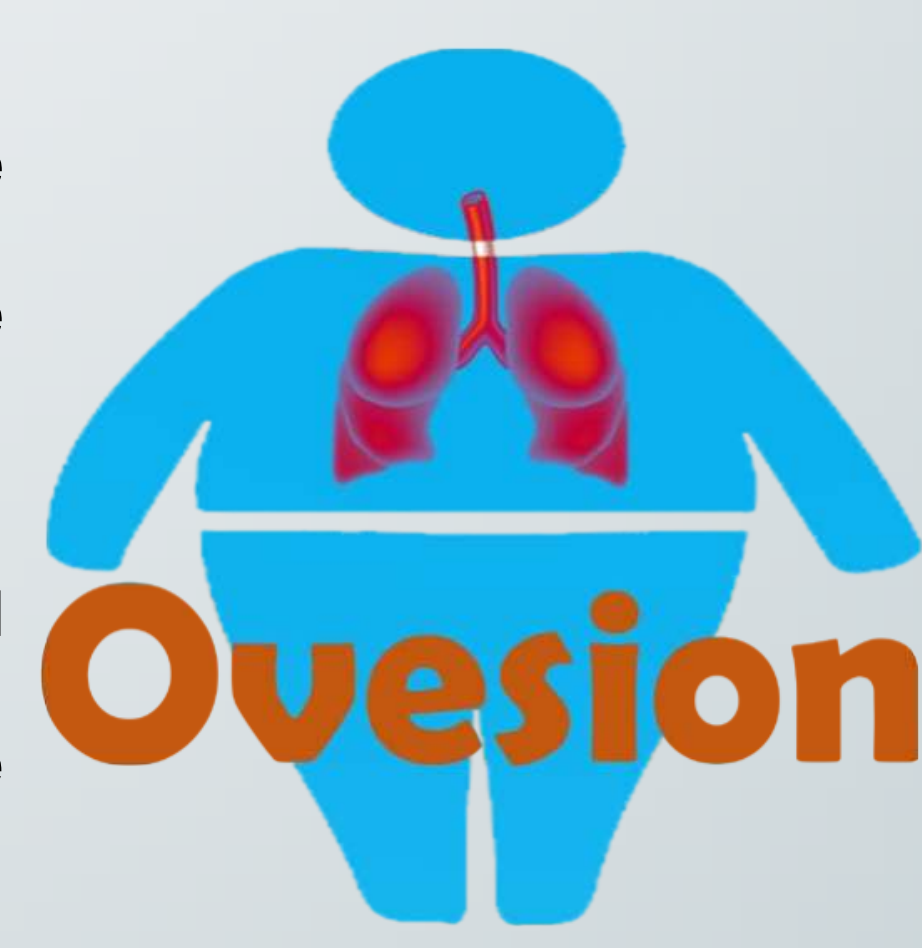

OvesionTT@gmail.com

\section{- Background and Goal of Study}

In obese patients' airway management is essential to prevent early arterial oxygen desaturation related to a reduced functional residual capacity $(F R C)$ and atelectasis formation during induction and after tracheal intubation (1).

The Macintosh laryngoscope is the standard method for endotracheal intubation. However, sometimes is ineffective and poorly tolerated by the obese patient.

The Totaltrack VLM ${ }^{\circledR}$ (TT VLM) (MedComflow S.A., Spain) is a hybrid device, between a supraglottic airway and a videolaryngoscope with an anatomically shaped blade (2).

However, despite its use in clinical practice, there are no comparative

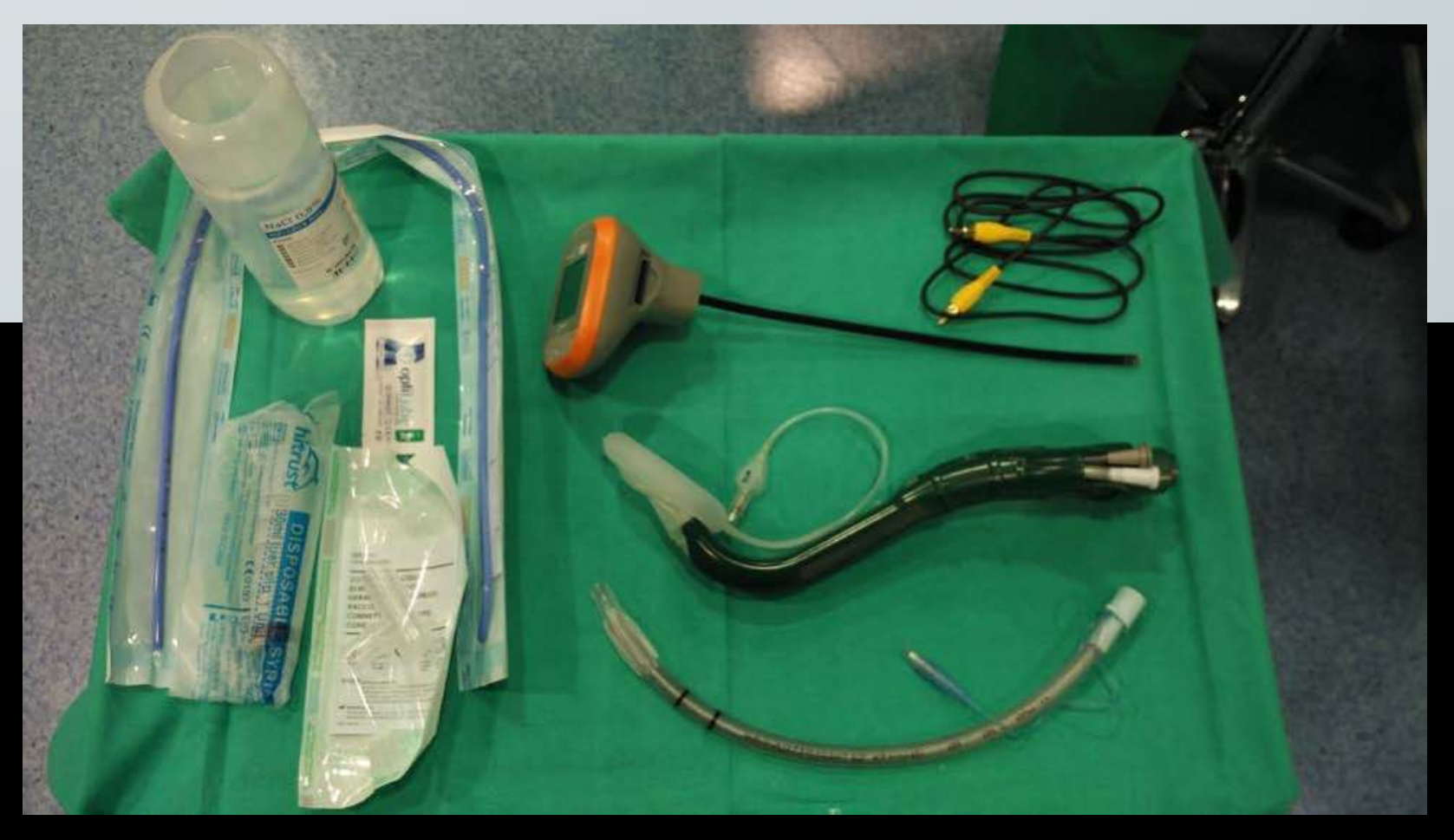
studies regarding direct laryngoscopy in obese patients.

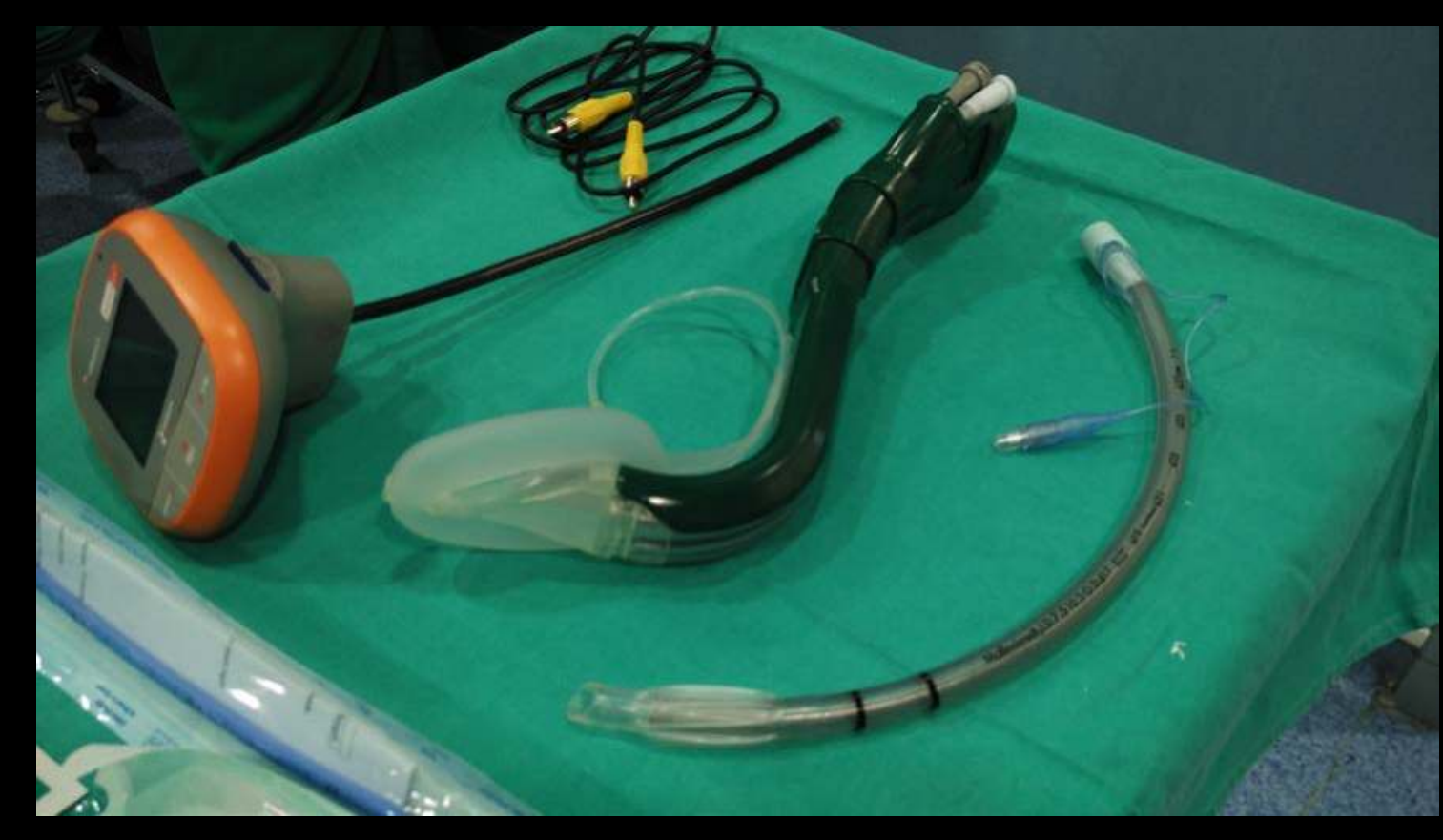

- Results and Discussion

In a retrospective 96 case-series analysis of hemodynamic (systolic and diastolic pressure) and ventilation efficiency (pulsioximetry) were measured before inserting the device in the mouth of the patient, and 1 and 5 minutes later. Patients mean BMI was 26, and ASA status was 2 in $84 \%$, and 3 in $17 \%$. There no exists important incidences in $77.5 \%$ patients, difficulties not avoided intubation, and successful ventilation after intubation was in $100 \%$ patients. $74 \%$ of researchers said they were satisfied, and $15 \%$ very satisfied (3).

\section{- References}

1. Benumof JL. Obesity, sleep apnea, the airway and anesthesia. Curr Opin Anaesthesiol 2004;17:21-30.

2. Gómez-Ríos MA, Freire-Vila E, Vizcaíno-Martínez L, et al. The Totaltrack ${ }^{\mathrm{TM}}$ : an initial evaluation. Br J Anaesth. 2015;115(5):798-799.

I. Hinojal Blanco et al. TOTALTRACK VLM ${ }^{\circledR}$, OUR CASE SERIES: NEW SOLUTIONS FOR OLD PROBLEMS. Trends in Anaesthesia and Critical Care 16 (2017) 27e39.
- Materials and Methods

OVESION is an interventional randomized clinical study (NCT03106974).

$>$ Primary Outcome Measure is saturation of blood oxygen at the end of orotracheal intubation, established with a curve of Capnography.

> Secondary Outcome Measures are total time of successful intubation, total time of successful intubation, number of maneuvers needed, IDS Scale, POGO Score, number of attempts of endotracheal intubation, hemodynamic response, degree of satisfaction of the researcher in a Visual Analogic Scale (0-5), adverse effects encountered during intubation.

$>$ Estimated enrollment is 1.440 adult patients.

$>$ Inclusion criteria are BMI >30, ASA 1-3, scheduled surgeries that require orotracheal intubation, general anesthesia with neuromuscular relaxation before intubation and patients who sign informed consent.

- Exclusion criteria are ASA 4, difficult airway already known, alterations of airway documented, allergies to device components and urgent surgery.

\section{- Conclusion(s)}

We believe TT VLM facilitates oxygenation during tracheal intubation, under safe conditions and uninterrupted ventilation. In cases of a failed intubation attempt, TT VLM can continue optimal ventilation/oxygenation while the operator thinks about the next step of the strategy.

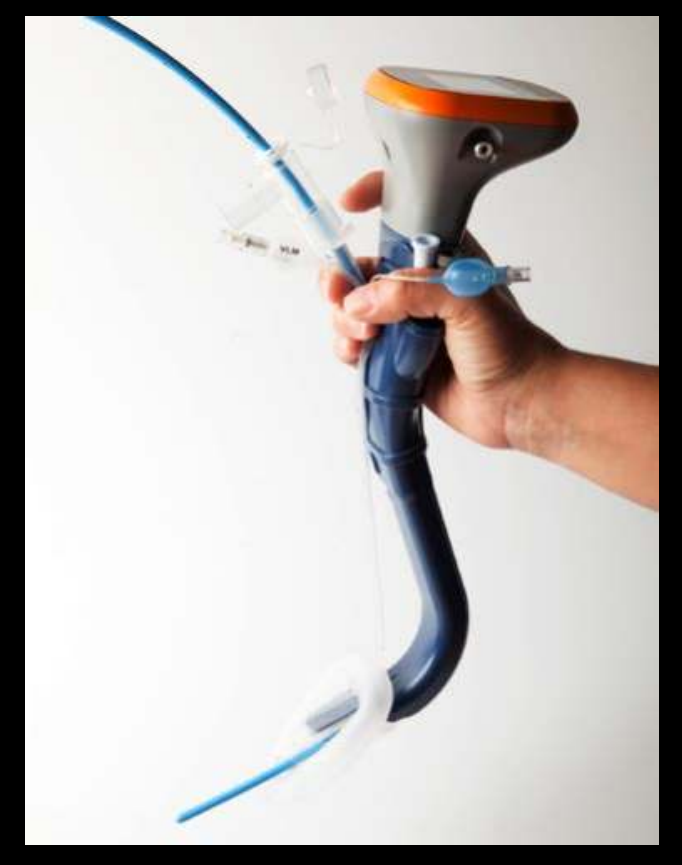

